\title{
Análisis del concepto de función en estudiantes sordos de grado décimo ${ }^{1}$
}

\section{Analysis of the concept of function in tenth grade deaf students}

\section{Análise do conceito de função em alunos surdos grau décimo}

Recibido: mayo de 2013

Aceptado: agosto de 2013
Raúl Peña Giraldo ${ }^{2}$

Eliécer Aldana Bermúdez ${ }^{3}$

\section{Resumen}

Este estudio de caso hace parte de una investigación que se está realizando con estudiantes sordos de grados octavo y décimo, con el propósito de lograr la comprensión/construcción del concepto de función, desde las dimensiones epistemológicas, didáctica y cognitiva. El estudio se fundamenta en el marco teórico de los registros de representación semiótica y la metodología de la Ingeniería didáctica, apoyado en el diseño, desarrollo e implementación de un software.

Palabras clave: Función; necesidades especiales; alumnos discapacitados; sordos; sistemas semióticos de representación, ingeniería didáctica; matemáticas escolares; álgebra; funciones.

\begin{abstract}
This case study is part of an investigation that is being conducted with deaf students in grades eight, and ten, in order to reach understanding / construction of the concept of function, since the dimensions epistemological, didactic and cognitive. The study is based on the theoretical framework of semiotic representation registers and methodology of teaching engineering, supported in the design, development and implementation of software.
\end{abstract}

Keywords: Function; special needs students with disabilities, deaf semiotic representation systems, engineering teaching, school mathematics, algebra functions.

\section{Resumo}

Este estudo de caso é parte de uma investigação que está sendo realizado com alunos surdos nas classes de oito e dez anos, a fim de chegar a um entendimento / construção do conceito de função, uma vez que as dimensões epistemológica, didática e cognitiva. $\mathrm{O}$ estudo baseia-se no quadro teórico de

1 Articulo de Investigación

2 Universidad del Quindio. Armenia, Colombia. Contacto: rpena@uniquindio.edu.co

3 Universidad del Quindio. Armenia, Colombia. Contacto: eliecerab@uniquindio.edu.co 
registros de representação semiótica e metodologia de ensino de engenharia, apoiada na concepção, desenvolvimento e implementação de software.

Palavras-chave: Função; alunos com necessidades especiais, com deficiência, sistemas de representação semiótica surdos, o ensino de engenharia, matemática escolar, funções de álgebra.

\section{Presentación del problema}

Existen numerosas investigaciones que evidencian las dificultades en el estudio del concepto de función, (González-Martín \& Camacho, 2005) señalan que algunas dificultades asociadas al concepto de función pueden deberse a la gran variedad de representaciones que éste tiene (gráfica, diagrama de flechas, fórmula, tabla, descripción verbal...) y las relaciones entre ella; sin embargo, el hecho de visualizar e integrar diferentes representaciones de un concepto no es algo que el estudiante realice automáticamente, sino que debe ser enseñado explícitamente. (Artigue, 1995) también cita que se han encontrado dificultades para articular los diferentes registros simbólicos de las expresiones de la noción de función y se han detectado dificultades en los estudiantes para identificar lo que en realidad es una función y la comprensión del concepto. En este sentido (García, Vázquez, \& Hinojosa, 2004), afirman que las tareas que implican la conversión del registro gráfico al algebraico son las que presentan mayor dificultad para los estudiantes. Las inquietudes planteadas en este trabajo van más allá de las dificultades enunciadas anteriormente, pues pretende realizar la investigación con estudiantes sordos. Este tipo de estudiantes no debe tener problemas en adquirir este concepto matemático si consideramos a la sordera como un problema fisiológico y no cognitivo. (Mora \& Parraguez, 2012).

\section{Marco referencial conceptual}

En este estudio asumimos los sistemas semióticos de representación de (Duval 2004, p. 25), quien afirma que "No es posible estudiar los fenómenos relativos al conocimiento sin recurrir a la noción de representación. [...] porque no hay conocimiento que un sujeto pueda movilizar sin una actividad de representación". El concepto de función implica múltiples representaciones (verbal, algebraica, diagrama, gráfica) y la correspondencia y articulación entre ellas. Además, al respecto se encuentran otros estudios con este tipo de población como los de (Calderón \& León 2007) investigaron sobre cómo generar en estudiantes sordos las condiciones para el desarrollo de las competencias cognitivas y comunicativas en los distintos campos de los saberes escolares, otro estudio de (León, Calderón \& Orjuela 2010), en el cual presentan un sistema de dispositivos didácticos para el análisis y evaluación con profesores de estudiantes sordos y oyentes; asimismo, (Guilombo 2011) sobre la búsqueda de materiales para la enseñanza de la geometría en escolares de educación básica.

\section{Metodología}

Esta investigación es de tipo cualitativa e interpretativa, en cuanto se trata de analizar un fenómeno social a partir de un conocimiento previo de una realidad educativa. El diseño metodológico se apoya en la Ingeniería Didáctica, que se sustenta en la teoría de situaciones didácticas (Brousseau, 1997) y la teoría de la transposición didáctica (Chevallard, 1991). En su proceso metodológico se distinguen cuatro fases: 1 . análisis preliminares, 2 . diseño y análisis a priori de las situaciones, 3. experimentación y 4. análisis a posteriori y evaluación. La implementación de esta ingeniería didáctica es pertinente para este estudio de investigación con poblaciones sordas, porque permite de manera flexible que el profesor investigador elabore sus propias secuencias didácticas de enseñanza para mediar de esta manera en la comprensión de los saberes matemáticos que pretende ayudar a construir en sus educandos, por ejemplo, Calderón \& León (2009) presentan un estudio de ingeniería didáctica como metodología de investigación del discurso en el aula de matemáticas, con una perspectivas de investigación del discurso en el aula. 


\section{Análisis de datos.}

El estudio se realizó con dos estudiantes sordos de grado décimo que asisten a clase de matemáticas con estudiantes oyentes en las mismas condiciones y que ya han estudiado la noción de función. El instrumento consistió en un taller exploratorio de los conceptos previos y los elementos que componen el concepto y fue aplicado con la apoyo de un intérprete de lengua de señas. En una de las actividades se pide al estudiante ubicar puntos en el plano cartesiano, y se pregunta si las parejas ordenadas $(3,2)$ y $(2,3)$ representan el mismo punto.

Grafico 1: Respuesta del estudiante 1

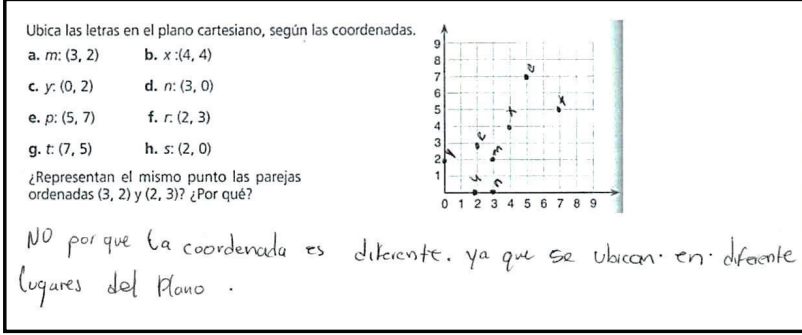

Fuente: Elaboración propia

Grafico 2: Respuesta del estudiante 2

\begin{tabular}{|c|c|c|}
\hline $\begin{array}{l}\text { ¿Representan el mismo punto las parejas } \\
\text { ordenadas }(3,2) \text { y }(2,3) \text { ? ¿Por qué? } \\
\text { no porque el Dunto esta ubicado en } \\
\text { ofra Cocosdenada aunque los numero }\end{array}$ & $\begin{array}{l}\underset{0123456789}{\stackrel{4}{2}} \\
\text { de la coordenada }\end{array}$ & rguat \\
\hline
\end{tabular}

Fuente: Elaboración propia

Los estudiantes ubican de manera correcta puntos en el plano y cuando se les invierte la abscisa y la ordenada ponen en evidencia que diferencian entre el eje $\mathrm{x}$ y el eje $\mathrm{y}$, haciendo uso de la palabra coordenada que hace parte lenguaje matemático.

La otra actividad consiste en determinar cuáles de los diagramas sagitales son función y cuáles no, los dos estudiantes seleccionan los mismos diagramas como funciones, y de acuerdo a la explicación escrita que dan y a la información dada al intérprete al realizar la actividad, se puede evidenciar que para ellos es función si todos los elementos del conjunto de partida están relacionados con algún elemento del conjunto de llegada y que todos los elementos del conjunto de llegada están relacionados con algún elemento del conjunto de partida, es decir, que todos los elementos deben tener pareja. Esto permite evidenciar que aunque determinan el conjunto de partida y el conjunto de llegada, no diferencian el dominio y el rango y para ellos es lo mismo.

Grafica 3: Respuesta del estudiante 1

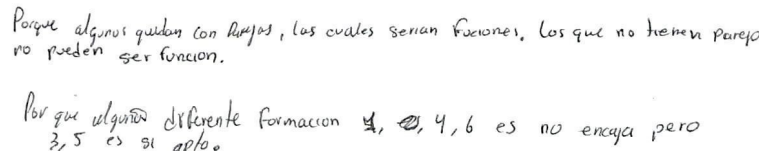

Fuente: Elaboración propia

Grafica 4: Respuesta del estudiante 2

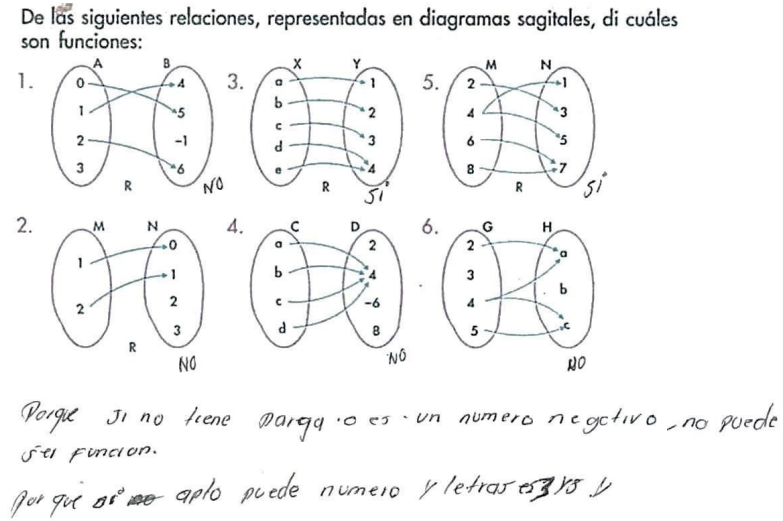

Fuente: Elaboración propia

Además, no saben que un elemento del dominio no puede tener dos imágenes ni que dos elementos del dominio pueden tener la misma imagen; lo importante es que todos los elementos del conjunto de partida hagan pareja con cualquier elemento del conjunto de llegada y que todos los elementos estén relacionados. Se puede pensar que la forma como determinan que es función está dado por creencias personales y análisis de los ejercicios propuestos más que por una comprensión del concepto. En cuanto a la pregunta ¿Recuerda usted qué es una función matemática? Podría explicar con sus propias palabras. Se apoyaron en el modo gráfico con diagramas sagitales, no utilizan las representaciones cartesianas y expresan que la representación algebraica les da dificultad para "trabajarla - entenderla". Con palabras propias contestaron:

Grafica 5: Respuesta del estudiante 1

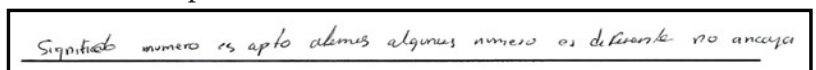

Grafica 6: Respuesta del estudiante 2

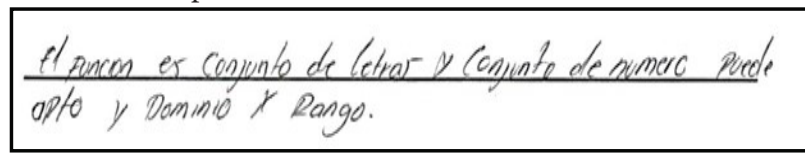

Fuente: Elaboración propia 
Al indagarles sobre lo que significaba las expresiones: apto y encaja, manifiestan que es una relación entre los dos conjuntos, y que les asusta y no entienden las expresiones algebraicas.

\section{Conclusiones}

Los estudiantes sordos ubican puntos en el plano, identifican y diferencian parejas ordenadas y establecen relaciones entre conjuntos; con el diseño de secuencias didácticas adecuadas se puede lograr la comprensión/construcción del concepto función; el registro de representación gráfico motiva a los no oyentes para lograr la articulación con los modos de representación algebraicas o verbales que les causa tanta dificultad, por tanto la visualización juega un papel importante en el estudio del concepto de función.

\section{Referencias}

Artigue, M. (1995). La enseñanza de los principios del cálculo: problemas epistemológicos, cognitivos y didácticos, en Ingeniería didáctica en educación matemática. México: Una empresa docente \& Grupo Editorial Iberoamericano.

Brousseau, G. (2004). Research in mathematical education, Regular Lecture en el 10th. International Congress on Mathematics Education (ICME10). Dinamarca.

Calderón, D. \& León, O. (2007). El Desarrollo de Competencia Comunicativa en Matemáticas en Estudiantes Sordos. Proyecto Colciencias.

Calderón, D., León, O. (2009). La ingeniería didáctica como metodología de investigación del discurso en el aula de matemáticas. En: perspectivas de la investigación del discurso en el aula. Publicación del Énfasis de Lenguaje del Doctorado Interinstitucional en Educación. Bogotá: Universidad Distrital Francisco José de Caldas. (En prensa).

Calderón, D. León, O. \& Orjuela, M. (2009). La relación lenguaje- matemáticas en la didáctica de los sistemas de numeración: aplicaciones en población sorda, Asocolme. Pasto.
Calderón, D. León, O. \& Orjuela, M. (2010). Didáctica de la cantidad para poblaciones sordas: los micromundos de la aproximación y de la precisión, Asocolme. Bogotá.

Duval, R. (2004). Semiosis y Pensamiento Humano Registros Semióticos y Apendizajes Intelectuales. Santiago de Cali: PeterLang S.A.

García, L., Vázquez, R. A., \& Hinojosa, M. (2004). Dificultades en el aprendizaje del concepto de función en estudiantes de ingeniería. Ingenierías, Revista de la Facultad de Ingeniería Mecánica y Eléctrica de la Universidad Autónoma de Nuevo León(24), 27-34.

Guilombo, M. (2011) La búsqueda de materiales para la enseñanza de la geometría con población sorda de primer grado de educación básica: un proceso de investigación. Universidad Distrital Francisco José de Caldas. Bogotá D.C.

González-Martín, A. S., \& Camacho, M. (2005). La generalización de la integral definida desde las perspectivas numérica, gráfica y simbólica utilizando entornos informáticos. Problemas de enseñanza y de aprendizaje. Tesis Doctoral, Universidad de la Laguna, San Cristóbal de La Laguna.

Mora, G., \& Parraguez, M. (2012). Estudio de la función lineal en estudiantes con déficit auditivo: ¿Un problema de tiempo o ritmo de aprendizaje? Unión, Revista Iberoamericana de Educación Matemática(31), 85-106.

Serrano Pau, C., \& Silvestre, N. (1995). Proceso de resolución de problemas aritméticos en el alumnado sordo: aspectos diferenciales respecto al oyente. Tesis Doctoral, Universidad Autonoma de Barcelona, Barcelona.

Van Lamoen, S., \& Parraguez, M. (2011). Construcción del conceptodefuncióncuadrática en estudiantes sordos. Acta Latinoamericana de Matemática Educativa(24), 331-339. 